\title{
Influence of surfactant and processing conditions in the stability of oil-in-water nanoemulsions
}

\author{
Hélder D. Silva *, Miguel A. Cerqueira, António A. Vicente \\ Centre of Biological Engineering, Universidade do Minho, Campus de Gualtar, 4710-057 Braga, Portugal
}

\section{A R T I C L E I N F O}

Article history:

Received 14 January 2015

Received in revised form 9 June 2015

Accepted 18 July 2015

Available online 31 July 2015

\section{Keywords:}

Nanoemulsion

Process conditions

Physical characterization

Medium chain triglycerides

Kinetic stability

\begin{abstract}
A B S T R A C T
This work evaluates the influence of the type of surfactant (Tween 20, SDS and DTAB) and processing conditions on the stability of oil-in-water nanoemulsions, measured in terms of hydrodynamic diameter $\left(H_{d}\right)$, polydispersity index (PdI) and zeta potential $(\mathrm{Zp})$. Nanoemulsions were prepared using high-pressure homogenization based on a $2^{4}$ level factorial design. Results show that processing parameters such as homogenization pressure, surfactant concentrations and oil:water ratio significantly affected the values of $H_{d}$ and PdI of nanoemulsions. The value of $H_{d}$ of anionic nanoemulsions decreased (from 177 to $128 \mathrm{~nm}$ ) with the increase of the homogenization pressure. The increase in the surfactant concentration and the decrease of the oil:water ratio lead to a decrease of $H_{d}$ for the cationic nanoemulsions (from 198 to $135 \mathrm{~nm}$ ). The increase of the oil:water ratio lead to a decrease of $H_{d}$ for the non-ionic nanoemulsions (from 341 to $171 \mathrm{~nm}$ ); this is contrary to the usual assumption that higher content in oil results in higher values of $H_{d}$. Those nanoemulsions showed a good kinetic stability (evaluated after centrifugation, heating-cooling cycles and thermal stress) upon measuring the $H_{d}$ during 28 and 35 days of storage, without visual evidence of creaming and phase separation. After one year of storage the nanoemulsions produced with the anionic surfactant remained kinetically stable, without visual evidence of creaming and/or phase separation.
\end{abstract}

(c) 2015 Elsevier Ltd. All rights reserved.

\section{Introduction}

Driven by consumers' demands for new and healthier food products the food industry seeks for new methodologies able to encapsulate, protect and release functional compounds. Based on this, researchers are focusing their efforts in relevant issues to food and nutrition regarding the improvement of food quality, through nanotechnology (Cerqueira et al., 2013; Silva et al., 2012).

Nanoemulsions are interesting for the food industry due to their potential applications as delivery systems of bioactive compounds while preventing their degradation and improving their bioavailability (Donsì et al., 2011; Guttoff et al., 2015; Silva et al., 2012). Nanoemulsions generally consist of lipid droplets between 10 and $200 \mathrm{~nm}$ dispersed in an aqueous phase, where surfactant molecules surround each oil droplet (Acosta, 2009; Cerqueira et al., 2014). Nanoemulsions can be produced using either low-energy or high-energy methods. Low-energy methods mainly depend on the intrinsic physicochemical properties of surfactants and oily phase, forming nanoemulsions by simple mixing procedures or by changing the system conditions such as temperature

\footnotetext{
* Corresponding author.

E-mail address: heldersilva@deb.uminho.pt (H.D. Silva).
}

or composition (Komaiko and McClements, 2015; Silva et al., 2012; Solans and Solé, 2012). High-energy methods make use of devices that apply high mechanical energy inputs to disrupt and combine the oil and water phases, forming small droplets (Abbas et al., 2013; Cerqueira et al., 2014; Komaiko and McClements, 2015; Silva et al., 2012). High-pressure homogenization is pointed as the most appropriate method for industrial applications, due to the facility of operation, scalability, reproducibility, and high throughput (Cerqueira et al., 2014; Donsì et al., 2011).

Preparing a nanoemulsion by a high-energy method implies using an oily and an aqueous phase, a surfactant and energy (Tadros et al., 2004; Walstra, 1993). The nano emulsification process by high-pressure homogenization comprises both the deformation and disruption of the droplets with the subsequent increase of the surface area and at the same time the droplet stabilization occurs by means of adsorption of the emulsifiers at the interface of the droplets (Donsì et al., 2011; Stang et al., 2001). The difference in the interfacial free energy between the initial and final state is by definition equal to the increase on the surface area between the oily and aqueous phases multiplied by the interfacial tension (McClements, 2005). The energy necessary to increase the interfacial area $(\gamma \Delta A)$ is very high and positive (i.e. it increases after homogenization), while the small entropy of the 
dispersion, and the corresponding energy $(T \Delta S)$ is also positive but cannot compensate the interfacial free energy (McClements, 2005; Schramm, 2006b; Tadros et al., 2004) and therefore Eq. (1) is always positive.

$\Delta G_{\text {formation }}=\gamma \Delta A-T \Delta S$

Thus nanoemulsion formation is always thermodynamically unfavorable, due to the increase of the interfacial area after emulsification and to the energy required to produce the droplets (McClements, 2005; Tadros et al., 2004; Walstra, 1993). In order to break up a droplet into smaller ones, it must be strongly deformed and this is opposed by the Laplace pressure, $\Delta p$, which is the difference in pressure between the inside and outside of the droplet, being the pressure greater on the inside of the droplet, given by:

$\Delta p=\gamma\left(1 / R_{1}+1 / R_{2}\right)$

where $R_{1}$ and $R_{2}$ are the principal radii of curvature of the droplet. For a nanoemulsion, a spherical droplet yields $R_{1}=R_{2}=R$ (Schramm, 2006b; Tadros et al., 2004; Walstra, 1993).

$\Delta p=2 \gamma / R$

Eq. (3) shows that the amount of energy needed to break the droplets increases when smaller droplets are produced; however, when lowering the interfacial tension $\Delta p$ is reduced and therefore the amount of energy needed to break up a droplet is reduced. Lowering the interfacial tension is one of the roles of the surfactants; nevertheless, their most essential role is preventing the coalescence of the newly formed droplets (Schramm, 2006b; Tadros et al., 2004; Walstra, 1993).

Surfactants preferentially adsorbs to the interfaces, once their molecular structures have non-polar hydrocarbon tails that favor non-polar liquids. Lowering the interfacial tension they will minimize the interfacial area between the continuous and dispersed phases and keep the interfaces smooth (Mason et al., 2006). Low molecular weight surfactants are able to decrease the interfacial tension in a greater extension than high molecular weight surfactants. This is mainly due differences in the orientation and configuration of the surfactants at the interface (Sari et al., 2015). Low molecular weight surfactants entirely adsorb and instantaneously orient themselves and the partitioning of the entire molecule between the two phases facilitates a maximum reduction in the interfacial tension (Sari et al., 2015). Also, a significant excess of surfactant in the continuous phase is needed this enables the new surface area of droplets to be quickly coated during emulsification, inhibiting disruption induced coalescence. This generally forms surfactant micelles that dissociate into monomers that rapidly adsorbs to the surface of the droplets (Mason et al., 2006; Rao and McClements, 2012). After adsorption of the surfactant to the surface of a droplet, surfactants most provide repulsive forces strong enough to prevent droplets aggregation. Ionic surfactants provide a great stability due electrostatic repulsions between droplets. Non-ionic surfactants provide stability due short-range repulsive forces, such as steric overlap, hydration, thermal fluctuation interactions, that prevents droplets from getting to close. Briefly, a surfactant must have three characteristics to be effective, first, rapidly adsorption to the surface of the new droplets; second, drastically reduce the interfacial tension and third form a membrane that prevents droplets from aggregating (McClements, 2002).

Commercial applications of nanoemulsions are one of the emerging fields of nanotechnology applied to food industry; the appearance in the food market of nanoemulsion-based food products has been growing in the last years. The application of nanoemulsions in the food industry can be subdivided into five major categories: fortified beverages, with NutraLease, NovaSol and SunActive Iron beverages, from NutraLease, AquaNova and High Vive company's, respectively (AquaNova, 2013a; High Vive, 2013; NutraLease, 2011); food colorants, with NovaSol BCS and Color Emulsion from AquaNova and Wild Flavours Inc, respectively (AquaNova, 2013b; Wild Flavors Inc., 2013); food packaging with BioNutriCoat from Improveat (Improveat, 2014); food supplements, with NanoResveratrol from Life Enhancement (Life Enhancement, 2013), Spray for Life from NanoSinergy (NanoSinergy, 2013) and NutriNano CoQ-10 from Solgar (Solgar, 2013); and fortified oils with Canova Active Oil from Shemen Industries (Shemen Industries, 2013).

One of the aims of this work was to study the effects of different charge surfactants, an anionic surfactant, Sodium Dodecyl Sulphate (SDS); a cationic surfactant, dodecyltrimethylammonium bromide (DTAB) and a non-ionic surfactant, Tween 20 (Pinheiro et al., 2013). The effect of the process conditions i.e., pressure, number of cycles, surfactant concentrations and oil content in the mean hydrodynamic diameter $\left(H_{d}\right)$, polydispersity index (PdI), zeta potential $(Z p)$ and the stability of the nanoemulsion were also evaluated. Furthermore, the theoretical minimum mean droplet diameter, creaming, the specific surface area and the energy dispended to produce the nanoemulsions were evaluated.

The surfactants used in this study were applied as model surfactants based on their different charge. Despite, they are commonly used in biotechnology and cosmetics industry, for EFSA, SDS and DTAB cannot be applied in foods, while Tween 20 is consider a food additive (EFSA, 2010). Nevertheless, considering FDA regulation SDS can be applied in food products as surfactant in fruit juice drinks under $25 \mathrm{ppm}$ and in coatings on fresh citrus fruit (FDA, 2014a, 2014b). Regarding DTAB, it can be used as an indirect food additives: adjuvants, production aids and sanitizers in contact with food products (FDA, 2014c).

\section{Materials and methods}

\subsection{Materials}

Neobee 1053 medium chain triglycerides (MCTs) is caprylic/capric triglyceride oil with a fatty acid distribution of 55\% of C8:0 and $44 \%$ of C10:0 was kindly provided by Stepan (The Netherlands) and was used without further purification. Tween 20 and sodium dodecyl sulphate (SDS) were purchased from Sigma-Aldrich (St. Louis, MO, USA) and dodecyltrimethylammonium bromide (DTAB) was acquired from Acros Organics (Geel, Belgium). Milli-Q water (Milli-Q apparatus, Millipore Corp., Bedford, MA, USA) was used to prepare all solutions.

\subsection{Experimental procedures}

2.2.1. Preparation of non-ionic, cationic and anionic nanoemulsions by high-pressure homogenization

Oil-in-water $(\mathrm{O} / \mathrm{W})$ emulsions were prepared according to (Pinheiro et al., 2013) with some modifications. Briefly, the nanoemulsions were pre-mixed during $2 \mathrm{~min}$ at $5000 \mathrm{rpm}$ using an Ultra-Turrax homogenizer ( $\mathrm{T}$ 25, Ika-Werke, Germany) followed by passage through a high-pressure homogenizer (Nano DeBEE, BEE International, USA) according to the fractional factorial design (Table 1 ). To assess the effect of operational conditions on emulsion stability at the nanoscale, samples were evaluated during a 28, 35 and 365 days of storage. The stability at day 365 was only performed for the nanoemulsions with the best stability results at day 35 . The prepared emulsions were stored at $4{ }^{\circ} \mathrm{C}$ in the absence of light, during the evaluation period. 
Table 1

Independent variables used in the $2^{4}$ fractional factorial design: pressure of homogenization (expressed in terms of Psi), number of cycles between each homogenization, surfactant concentration (expressed in \% wt) and oil to water $(\mathrm{O} / \mathrm{W})$ volume ratio (expressed in \% vol)

\begin{tabular}{clllr}
\hline Sample & Pressure $($ Psi $* 1000)$ & Cycles & Surfactant & O/W ratio (\% vol) \\
\hline 1 & 10 & 10 & 0.5 & 5 \\
2 & 20 & 10 & 0.5 & 15 \\
3 & 10 & 30 & 0.5 & 15 \\
4 & 20 & 30 & 0.5 & 5 \\
5 & 10 & 10 & 1.5 & 15 \\
6 & 20 & 10 & 1.5 & 5 \\
7 & 10 & 30 & 1.5 & 5 \\
8 & 20 & 30 & 1.5 & 15 \\
9 & 15 & 20 & 1 & 10 \\
10 & 15 & 20 & 1 & 10 \\
11 & 15 & 20 & 1 & 10 \\
\hline
\end{tabular}

\subsubsection{Kinetic stability studies}

Kinetic stability studies were performed through centrifugation, heating-cooling cycle and thermal stress as followed:

Centrifugation: formulations were centrifuged at $5000 \mathrm{~g}$ for $30 \mathrm{~min}$. Those formulations that did not show any phase separation were taken for the heating-cooling cycle test.

Heating-cooling cycle: formulations were subjected to six cycles between $4{ }^{\circ} \mathrm{C}$ and $45^{\circ} \mathrm{C}$ with a storage time higher than $48 \mathrm{~h}$ at each temperature. Those formulations, which were stable at these temperatures cycles, were further taken for size measurements (Shafiq et al., 2007).

Thermal stress: The influence of temperature on nanoemulsions stability was examined through Dynamic Light Scattering (DLS) technique, by measuring the $H_{d}$ of the nanoemulsions. Briefly, $1 \mathrm{~mL}$ of the formulations were submitted to a range of temperatures from 20 to $80^{\circ} \mathrm{C}$, with increasing intervals of $10^{\circ} \mathrm{C}$, during $10 \mathrm{~min}$ at each condition (Morais Diane and Burgess, 2014). The temperature was adjusted using a Nano ZS-90 equipment (Zetasizer Nano ZS-90, Malvern Instruments, Worcestershire, UK). The data was reported as the mean droplet diameter (hydrodynamic diameter, $\left.H_{d}\right)$, mean $\pm \mathrm{SD}(n=3)$.

\subsubsection{Nanoemulsion size measurements}

The particle size distribution and Polydispersity Index (PdI) of nanoemulsions were determined using DLS (Zetasizer Nano ZS-90, Malvern Instruments, Worcestershire, UK). The nanoemulsion samples were diluted $10 \times$ in distilled water at ambient temperature. The data was reported as the mean droplet diameter (hydrodynamic diameter, $H_{d}$ ). Polydispersity Index is a dimensionless and indicates the heterogeneity (monodisperse or polydisperse) of sizes of particles in a mixture (Malvern, 2011). Each sample was analyzed in a disposable polystyrene cell (DTS0012, Malvern Instruments). The measurements were performed in duplicate, with three readings for each of them. The results are given as the average \pm standard deviation of the six values obtained (Rao and McClements, 2013; Silva et al., 2011).

\subsubsection{Nanoemulsion charge measurements}

The droplet charge (zeta potential) of the nanoemulsions was determined using a particle micro-electrophoresis instrument (Zetasizer Nano ZS-90, Malvern Instruments, Worcestershire, UK). Samples were diluted $100 \times$ in distilled water prior to measurements in order to avoid multiple scattering effects at ambient temperature and placing the diluted emulsions into disposable capillary cells (DTS 1060, Malvern Instruments) (Ozturk et al., 2014; Rao and McClements, 2013).

\subsubsection{Nanoemulsion creaming rate}

Ten grams of nanoemulsion were transferred into a $15 \mathrm{~mL}$ conical centrifuge tube (Falcon ${ }^{\mathrm{TM}}$, Fisher Scientific), tightly sealed with a plastic cap, and then stored at $4{ }^{\circ} \mathrm{C}$ ) for approximately $24 \mathrm{~h}, 1$ month, 3 months and 1 year. After storage, several emulsions separated into an opaque layer at the top, a turbid layer in the middle, and a transparent layer at the bottom. The "serum layer" was defined to be the sum of the turbid and transparent layers. The total height of the emulsion (HE) and the height of the serum layer $(H S)$ were measured using a ruler ( $\mathrm{Li}$ et al., 2010).

\subsubsection{Microscopy}

The morphology of nanoemulsions was evaluated by transmission electron microscopy (TEM) (EM 902A, ZEISS, Germany) operating at $80 \mathrm{kV}$. TEM samples were prepared by depositing the nanoemulsion suspensions on a carbon-coated copper grid, and negatively stained with $1 \%(\mathrm{w} / \mathrm{v})$ uranyl acetate for observation. Samples were air-dried before analyses.

\subsubsection{Nanoemulsions viscosity measurements}

Viscosity measurements of the nanoemulsion samples were conducted using a dynamic shear rheometer (TA Instruments, New Castle, DE) with a shear rate profile from 0.1 to $1000 \mathrm{~s}^{-1}$. All the measurements were performed at $25^{\circ} \mathrm{C}$. The values of these measurements were applied to Eqs. (7) and (8).

\subsubsection{Nanoemulsions density measurements}

Density measurements of the nanoemulsion samples were conducted using a density-meter Densito 30PX (Mettler-Toledo, Inc., Columbus, $\mathrm{OH}$ ) at $25^{\circ} \mathrm{C}$. The values of these measurements were applied to Eqs. (6)-(8).

\subsubsection{Nanoemulsions interfacial tension measurements}

The interfacial tension of the nanoemulsions solution was measured by the pendant drop method using the Laplace-Young approximation, (Song and Springer, 1996), with a face contact angle meter (OCA 20, Dataphysics, Germany). The samples of the coatings were taken with a $500 \mu \mathrm{L}$ syringe (Hamilton, Switzerland), with a needle of $0.75 \mathrm{~mm}$ of diameter. Three replicates were performed at $20^{\circ} \mathrm{C}$. The values of these measurements were applied to Eqs. (1)-(3).

\subsection{Statistical procedures}

\subsubsection{Experimental design}

A multifactor model ( $2^{4}$ fractional factorial design) was used to evaluate the independent variables (see Table 1 ): homogenization pressure (10,000-20,000 Psi), surfactant concentration $(0.5-1.5 \%$ $\mathrm{w} / \mathrm{w})$, ratio between oily and water phase (O/W ratio) $(5-15 \%$ $\mathrm{v} / \mathrm{v}$ ) and cycles between each homogenization (10-30) and evaluate the main effects and interaction effects of these formulations on the hydrodynamic diameter, polydispersity index and zeta potential. A total of 11 experiments for each surfactant were conducted with 3 center points (in order to allow the estimation of pure error).

\subsubsection{Data analyses}

Data analyses were performed using Microsoft Windows Excel 2011, using the Tukey's Multiple Comparison Test with a confidence interval of 95\% in GraphPad Prism 5 (GraphPad Software, Inc.) and using ANOVA in STATISTICA 7.0 (Statsoft, Tulsa, OK, USA). 


\section{Results}

\subsection{Effect of process conditions on nanoemulsions}

The combination of surfactant and a high-energy homogenization process (using an Ultra-Turrax as a premix step followed by a high-pressure homogenization process) allowed the formation of nanoemulsions with different characteristics. For an initial screening, the hydrodynamic diameter $\left(H_{d}\right)$, the polydispersity index (PdI) and the zeta potential $(Z p)$ of nanoemulsions are the most studied parameters, once they give an accurate idea about the formed nanoemulsions and their stability (Cheong et al., 2008; Sood et al., 2014; Tan and Nakajima, 2005).

Table 2 shows the values of $H_{d}$ for the nanoemulsions produced with Tween 20, SDS and DTAB. Values ranged between 171 and $341 \mathrm{~nm}$ for nanoemulsions produced with Tween 20; between 126 and $177 \mathrm{~nm}$ when produced with SDS and between 135 and $198 \mathrm{~nm}$ for DTAB. Transmission Electron Microscopy (TEM) confirmed the development of these nanoemulsions and validated the mean droplet diameters achieved; Fig. 1 represents a TEM microphotograph of the anionic, cationic and non-ionic nanoemulsions produced using the high-pressure homogenization technique at $1 \%$ wt of surfactant, dispersed in the aqueous phase, and MCTs oil as the organic phase at 15,000 Psi, during 20 cycles. Also, TEM was performed to observe the morphology of the different charge nanoemulsions. In Fig. 1a it is possible to see oil-droplet aggregations due dehydration conditions after the use of uracyl.

In order to study the most influent factors on the dependent parameters $\left(H_{d}\right.$, PdI and $\left.Z p\right)$ a multifactor model $2^{4}$ was used, being the results analyzed using ANOVA, which identifies the significant factors that affect the responses (Table 3 ). Results show that $H_{d}$ is significantly influenced $(p<0.05)$ by the homogenization pressure when SDS is used as surfactant; nevertheless, when DTAB was used as surfactants for the nanoemulsion production, $H_{d}$ is significantly influenced $(p<0.05)$ by homogenization pressure, surfactant concentration and $\mathrm{O} / \mathrm{W}$ ratio. In the case of nanoemulsions produced with Tween $20, H_{d}$ is significantly influenced $(p<0.05)$ by homogenization pressure, the interaction between the homogenization pressure and the $\mathrm{O} / \mathrm{W}$ ratio, by the surfactant concentration and the number of cycles. The nanoemulsions produced with SDS achieved the smallest value of $H_{d}$, followed by the nanoemulsions produced with DTAB and Tween 20 , respectively. The differences in $H_{d}$ values for the three surfactants used can be attributed to the molecular weight $\left(M_{w}\right)$ of the surfactants $\left(M_{w} \quad\right.$ SDS $<M_{w}$ $\mathrm{DTAB}<M_{w}$ Tween 20), in general small molecule surfactants can lead, under similar processing conditions, to smaller $H_{d}$ values, which has been attributed to differences in adsorption rates (faster adsorption rates result in smaller sizes) and in interfacial properties (such as thickness, charge, permeability, and environmental responsiveness) (Donsì et al., 2011; McClements, 2010; Qian and
McClements, 2011). Also, lowering the interfacial tension through the use of surfactants may facilitate droplet deformation and disruption (Brösel and Schubert, 1999).

As shown in Table 2 the mean $H_{d}$ of nanoemulsions decreases with the increase of homogenization pressure and number of cycles. This behavior is explained by the high intensity of shear forces produced during the homogenization process (Tan and Nakajima, 2005). Also, increasing the homogenization pressure and the number of cycles will lead to a higher specific energy, $E v$ (Eq. (4)), delivered to the emulsion by the high-pressure homogenizer, which is the mechanical energy input per unit of volume of the chamber where the droplets are disrupted. $E v$ can be calculated by the homogenization pressure $(\Delta P)$ times the number of cycles (n) (Donsì et al., 2011; Stang et al., 2001). This indicates that further increases of the dispersion energy levels lead to even smaller nanoemulsions (Donsì et al., 2011; Silva et al., 2011).

$E_{v}=\Delta P * n$

Tables 2 and 3 show that the increase of surfactant concentrations leads to lower values of $H_{d}$. This is due to the fact that more surfactant is present to cover any new droplet surfaces formed during homogenization and because the droplet surfaces will be covered more quickly by the surfactants (Qian and McClements, 2011). Also, higher surfactant concentrations increase the interfacial area, while reducing the interfacial tension, leading to lower droplet sizes (Anton et al., 2007; Esquena et al., 2003; Liu et al., 2006).

Table 2 shows the PdI values obtained for nanoemulsions, ranging from 0.164 to $0.242,0.119$ to 0.179 and 0.146 to 0.202 for Tween 20, SDS and DTAB, respectively. The efficiency of droplet disruption increased for higher specific energy (i.e. homogenization pressure times the number of cycles), at the most severe conditions the smallest mean droplet diameter was achieved, nevertheless for the PdI this did not happen. This is due the fact that PdI may also depend on emulsifier surface and dynamic properties (Donsì et al., 2011). Donsì et al. (2011) also verified that PdI was mainly affected by the kinetics of surface adsorption of the emulsifier.

ANOVA results show that the homogenization pressure significantly influenced $(p<0.05)$ the PdI values of nanoemulsions produced with the different surfactants (SDS, DTAB and Tween 20), as shown in Table 3. For the nanoemulsions produced with Tween 20 the interaction between pressure and $\mathrm{O} / \mathrm{W}$ ratio and the interaction between pressure and the number of cycles also significantly influenced $(p<0.05)$ PdI values. Regarding the nanoemulsions produced with DTAB, the interaction between pressure and $\mathrm{O} / \mathrm{W}$ ratio and the interaction between pressure and the number of cycles also significantly influenced $(p<0.05)$ PdI, as also did the number of cycles $(p<0.05)$.

Table 2

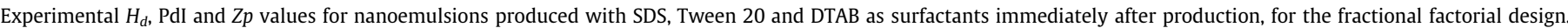

\begin{tabular}{|c|c|c|c|c|c|c|c|c|c|}
\hline \multirow[t]{2}{*}{ Sample } & \multicolumn{3}{|l|}{ SDS day 0} & \multicolumn{3}{|l|}{ DTAB day 0} & \multicolumn{3}{|c|}{ Tween 20 day 0} \\
\hline & $H_{d}(\mathrm{~nm})$ & $Z p(\mathrm{mV})$ & PdI & $H_{d}(\mathrm{~nm})$ & $Z p(\mathrm{mV})$ & PdI & $H_{d}(\mathrm{~nm})$ & $Z p(\mathrm{mV})$ & PdI \\
\hline 1 & $168.6 \pm 1.1$ & $-69.4 \pm 1.2$ & $0.135 \pm 0.01$ & $174.9 \pm 5.0$ & $64.8 \pm 0.3$ & $0.158 \pm 0.01$ & $257.8 \pm 2.7$ & $-15.3 \pm 1.0$ & $0.242 \pm 0.01$ \\
\hline 2 & $141 \pm 4.6$ & $-66.2 \pm 1.8$ & $0.150 \pm 0.02$ & $197.6 \pm 5.5$ & $43.0 \pm 0.5$ & $0.195 \pm 0.02$ & $171.2 \pm 4.6$ & $-30.1 \pm 4.8$ & $0.164 \pm 0.01$ \\
\hline 3 & $147.7 \pm 3.5$ & $-69.3 \pm 1.2$ & $0.131 \pm 0.01$ & $187 \pm 5.6$ & $43.1 \pm 2.4$ & $0.202 \pm 0.01$ & $341.2 \pm 8.1$ & $-23.1 \pm 0.7$ & $0.224 \pm 0.02$ \\
\hline 4 & $128.9 \pm 3.7$ & $-78.7 \pm 1.7$ & $0.153 \pm 0.01$ & $172.4 \pm 4.8$ & $71.0 \pm 1.2$ & $0.147 \pm 0.01$ & $323.3 \pm 4.7$ & $-16.6 \pm 3.5$ & $0.210 \pm 0.01$ \\
\hline 5 & $177.1 \pm 3.9$ & $-73.1 \pm 2.3$ & $0.179 \pm 0.02$ & $163.0 \pm 4.7$ & $71.2 \pm 2.5$ & $0.148 \pm 0.01$ & $277.9 \pm 8.8$ & $-25.0 \pm 0.5$ & $0.236 \pm 0.01$ \\
\hline 6 & $136.5 \pm 5.3$ & $-68.2 \pm 1.9$ & $0.130 \pm 0.01$ & $141.2 \pm 3.4$ & $70.3 \pm 2.9$ & $0.146 \pm 0.01$ & $256.2 \pm 10.5$ & $-20.1 \pm 2.4$ & $0.181 \pm 0.02$ \\
\hline 7 & $157.5 \pm 3.6$ & $-84.2 \pm 0.4$ & $0.119 \pm 0.01$ & $134.9 \pm 2.9$ & $73.1 \pm 3.1$ & $0.171 \pm 0.01$ & $215.5 \pm 6.1$ & $-27.5 \pm 1.0$ & $0.177 \pm 0.03$ \\
\hline 8 & $126.6 \pm 3.8$ & $-73.7 \pm 2.2$ & $0.148 \pm 0.02$ & $153.9 \pm 4.8$ & $74.6 \pm 2.2$ & $0.179 \pm 0.01$ & $195.8 \pm 5.8$ & $-20.4 \pm 2.2$ & $0.197 \pm 0.01$ \\
\hline $\mathrm{CP}$ & $140.6 \pm 12.3$ & $-73.5 \pm 3.8$ & $0.145 \pm 0.02$ & $156.3 \pm 2.7$ & $75.7 \pm 0.5$ & $0.197 \pm 0.02$ & $237.4 \pm 6.4$ & $-24.7 \pm 2.0$ & $0.192 \pm 0.01$ \\
\hline
\end{tabular}

Each value represents mean $\pm \mathrm{SD}(n=3)$; CP - central point; $H_{d}$ - hydrodynamic diameter; $Z p$ - zeta potential; PdI - polydispersity index. 


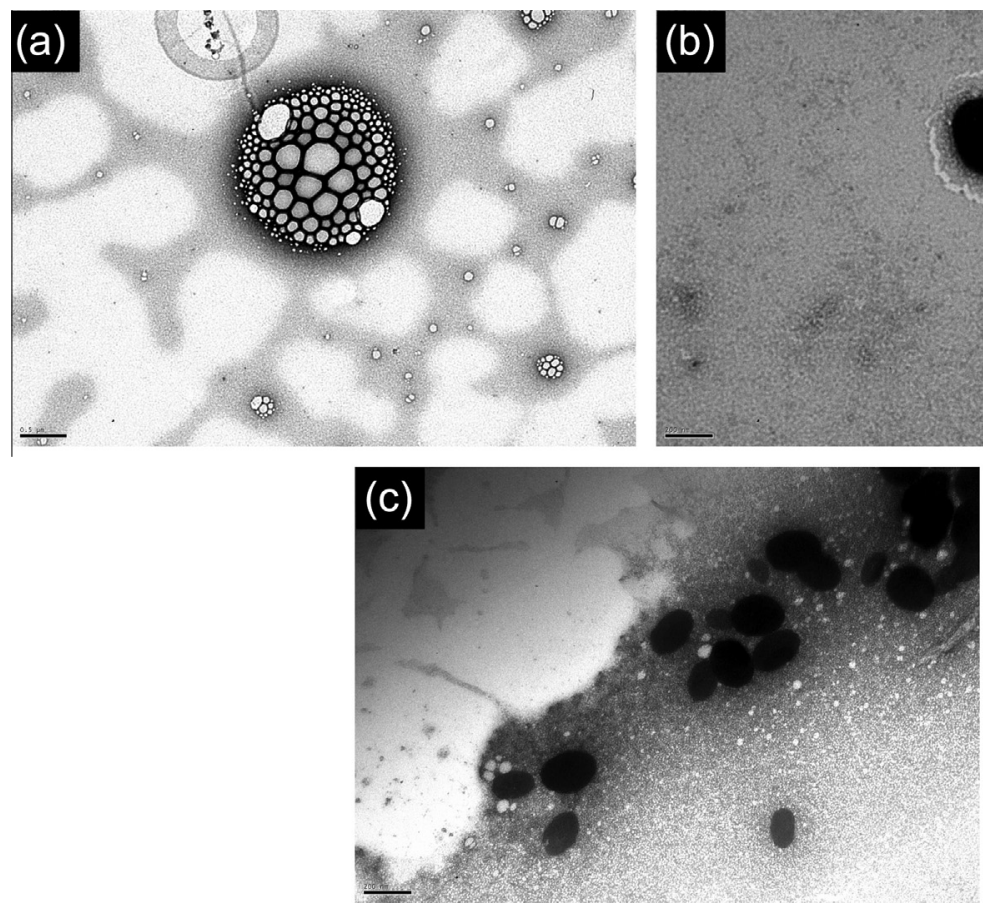

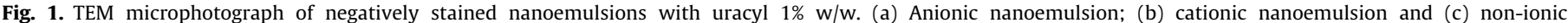
nanoemulsion.

Table 3

ANOVA results for dependent parameters estimation.

\begin{tabular}{|c|c|c|c|c|}
\hline & Independent variable & $\begin{array}{l}p \text {-value } \\
\text { SDS }\end{array}$ & $\begin{array}{l}p \text {-value } \\
\text { Tween }\end{array}$ & $\begin{array}{l}p \text {-value } \\
\text { DTAB }\end{array}$ \\
\hline \multirow[t]{5}{*}{$H_{d}$} & Pressure & 0.047301 & 0.014975 & 0.028920 \\
\hline & Pressure by $\mathrm{O} / \mathrm{W}$ ratio & - & 0.002528 & - \\
\hline & $\begin{array}{l}\text { Surfactant } \\
\text { concentration }\end{array}$ & - & 0.014530 & 0.004790 \\
\hline & Cycles & - & 0.024731 & - \\
\hline & Ratio & - & - & 0.013720 \\
\hline \multirow[t]{4}{*}{ PdI } & Pressure & 0.048942 & 0.013272 & 0.030854 \\
\hline & Pressure by $\mathrm{O} / \mathrm{W}$ ratio & - & 0.040542 & 0.010941 \\
\hline & Pressure by cycles & - & 0.011017 & 0.022267 \\
\hline & Cycles & - & - & 0.009264 \\
\hline \multirow[t]{2}{*}{$Z p$} & Pressure & 0.041880 & - & - \\
\hline & Pressure by cycles & - & 0.049921 & - \\
\hline
\end{tabular}

The influence of processing conditions on $Z p$ was also evaluated, being of extremely importance due to the different charges of the surfactants used. $Z p$ values ranged from -15 to $-30 \mathrm{mV}$ for nanoemulsions produced with Tween 20 , a non-ionic surfactant. The high values of $Z p$ obtained for the nanoemulsions produced with Tween 20 may be explained by the composition of the MCTs oil (free fatty acids and salts) and by the presence of MCTs oil at the interface of the surfactant, even at equilibrium, there is a continuous exchange of molecules between the interior of the droplets, the continuous phase and the interface, that will occur at a rate dependent of the mass transport of the molecules through the system (McClements, 2005). For nanoemulsions produced with SDS, an anionic surfactant, $Z p$ values ranged between -66 and $-84 \mathrm{mV}$. The $Z p$ of the nanoemulsion has a negative value due to the presence of head sulfate groups of SDS molecules (Jadhav et al., 2015). Nanoemulsions produced with DTAB (cationic surfactant) displayed $Z p$ values ranging from 43 to $76 \mathrm{mV}$. ANOVA results showed that the process parameters did not significantly influenced $Z p$ for all the developed nanoemulsions (Table 3). These electrical charges were expected as Tween 20, SDS and DTAB are non-ionic, anionic and cationic emulsifiers, respectively (Pinheiro et al., 2013).

\subsection{Kinetic stability of nanoemulsions during storage}

It is important to distinguish thermodynamic stability from kinetic stability; while thermodynamics will tell if a process will or not occur (no matter the time to complete it), kinetics will tell the rate (what time scale) and the degree of change, if it occurs. Kinetic stability tests were carried out to eliminate any unstable formulation, due to creaming or sedimentation. Tests were performed in terms of centrifugation and through heating and cooling cycles. Only those formulations that were found to be stable were selected for evaluation of $H_{d}$, PdI and $Z p$ during storage (Table 4).

\subsubsection{Size distribution}

Regarding centrifugation tests, all produced formulations (using all type of surfactants) showed no macroscopic instability phenomena (i.e. creaming or phase separation), and the sizes remained unchanged after centrifugation (data not shown), due to this all the formulations were subjected to the heating-cooling cycles. Once more the produced nanoemulsions showed no macroscopic instability phenomena (i.e. creaming or phase separation) and the sizes remained unchanged (data not shown) after the heating-cooling cycles' tests. Therefore, all formulations were evaluated by means of size stability during 28 days of storage, in order to evaluate the influence of the process conditions in the mean droplet diameter during storage.

Table 4 shows the values of $H_{d}$, PdI and $Z p$ of nanoemulsions after 28 days of storage. In general, it can be observed that these parameters remain unchanged after 28 days of storage. For the nanoemulsions produced with the anionic surfactant, SDS, only sample 5 shows an increase in size of 13 nm. Donsì et al. (2011) showed that SDS as a significantly short characteristic time for adsorbing to the oil-in-water interface, when compared to surfactants like polysorbates (e.g. Tween 20) (Donsì et al., 2011). Fast adsorption kinetics of the surfactant to the interface can 
Table 4

Experimental $H_{d}$ values obtained for nanoemulsions produced with SDS, Tween 20 and DTAB as surfactants after 28 days of storage for the fractional factorial design.

\begin{tabular}{|c|c|c|c|c|c|c|c|c|c|}
\hline \multirow[t]{2}{*}{ Sample } & \multicolumn{3}{|l|}{ SDS day 28} & \multicolumn{3}{|c|}{ DTAB day 28} & \multicolumn{3}{|c|}{ Tween 20 day 28} \\
\hline & $H_{d}(\mathrm{~nm})$ & $Z p(\mathrm{mV})$ & PdI & $H_{d}(\mathrm{~nm})$ & $Z p(\mathrm{mV})$ & PdI & $H_{d}(\mathrm{~nm})$ & $Z p(\mathrm{mV})$ & PdI \\
\hline 1 & $159.8 \pm 4.4$ & $-61.2 \pm 1.1$ & $0.136 \pm 0.03$ & $172.0 \pm 5.1$ & $63.1 \pm 0.8$ & $0.168 \pm 0.01$ & $174.2 \pm 2.4$ & $-14.7 \pm 0.1$ & $0.194 \pm 0.01$ \\
\hline 2 & $137.8 \pm 3.0$ & $-62.2 \pm 1.3$ & $0.141 \pm 0.01$ & $175.7 \pm 1.4$ & $58.3 \pm 0.6$ & $0.174 \pm 0.01$ & $166.1 \pm 4.5$ & $-32.3 \pm 0.3$ & $0.134 \pm 0.01$ \\
\hline 3 & $147.4 \pm 3.8$ & $-62.3 \pm 1.9$ & $0.121 \pm 0.01$ & $194.8 \pm 4.2$ & $60.7 \pm 0.9$ & $0.185 \pm 0.02$ & $195.5 \pm 6.8$ & $-22.8 \pm 1.1$ & $0.197 \pm 0.01$ \\
\hline 4 & $130.9 \pm 4.6$ & $-94.4 \pm 1.9$ & $0.165 \pm 0.01$ & $168.2 \pm 2.0$ & $67.0 \pm 0.7$ & $0.162 \pm 0.01$ & $253.9 \pm 7.4$ & $-15.1 \pm 0.7$ & $0.213 \pm 0.01$ \\
\hline 5 & $164.6 \pm 6.9$ & $-63.5 \pm 1.7$ & $0.168 \pm 0.03$ & $159.9 \pm 4.8$ & $66.3 \pm 1.9$ & $0.156 \pm 0.01$ & $203.6 \pm 3.7$ & $-27.8 \pm 0.4$ & $0.131 \pm 0.02$ \\
\hline 6 & $132.58 \pm 2.6$ & $-76.7 \pm 2.3$ & $0.141 \pm 0.02$ & $118.9 \pm 1.5$ & $76.8 \pm 1.5$ & $0.155 \pm 0.02$ & $245.9 \pm 5.2$ & $-19.5 \pm 0.2$ & $0.173 \pm 0.01$ \\
\hline 7 & $159.4 \pm 6.5$ & $-62.5 \pm 1.0$ & $0.138 \pm 0.01$ & $142.9 \pm 4.4$ & $70.8 \pm 1.9$ & $0.227 \pm 0.03$ & $177.8 \pm 4.2$ & $-24.3 \pm 0.3$ & $0.140 \pm 0.01$ \\
\hline 8 & $126.1 \pm 5.2$ & $-92.6 \pm 1.8$ & $0.139 \pm 0.01$ & $152.0 \pm 3.1$ & $74.9 \pm 1.0$ & $0.187 \pm 0.01$ & $196.7 \pm 0.2$ & $-25.7 \pm 2.3$ & $0.186 \pm 0.01$ \\
\hline $\mathrm{CP}$ & $140.5 \pm 4.0$ & $-78.2 \pm 1.4$ & $0.155 \pm 0.01$ & $155.4 \pm 3.0$ & $73.6 \pm 1.9$ & $0.196 \pm 0.01$ & $204.6 \pm 3.7$ & $-26.3 \pm 1.3$ & $0.125 \pm 0.01$ \\
\hline
\end{tabular}

Each value represents mean \pm SD $(n=3)$; CP - central point; Hd - hydrodynamic diameter; Zp - zeta potential; PdI - polydispersity index.

significantly reduce the re-coalescence phenomena. This conjugated with the lower oil-in-water interfacial tension lead to stable nanoemulsions with size values ranged between 130 and $165 \mathrm{~nm}$, depending on the processing conditions (Donsì et al., 2011).

The same behavior was observed for nanoemulsions produced with DTAB (cationic surfactant), where the size values remained unchangeable during storage time. Li and McClements (2011) prepared stable nanoemulsions using DTAB, achieving size values of approximately $187 \mathrm{~nm}$, indicating that these nanoemulsions were not susceptible to droplet aggregation; those authors also claim that the presence of DTAB in low concentrations increased the rate and extent the lipid digestion. One possible explanation is the fact that this surfactant may facilitate the adsorption of lipase to the nanoemulsion surface due to electrostatic attraction ( $\mathrm{Li}$ and McClements, 2011). For nanoemulsions produced with the non-ionic surfactant, Tween 20 , an increase in the size values of samples 1, 3, 4, 5 and 7 after storage was observed. This increase could be explained by the breakdown process of the emulsions, namely the creaming effect, where the larger droplets move faster to the top of the container, due the fact that when the gravitational forces exceed the thermal motion of the droplets (Brownian motion) a concentration gradient is built up (Tadros, 2013). Silva et al. (2011) also found a similar behavior for nanoemulsions produced with Tween 20 . They showed that the samples with lower size values had a greater tendency to aggregate, since they were more susceptible to Brownian motion, which leads to a superior chance of collision, allowing aggregation to become a dominant mechanism for emulsion instability (Silva et al., 2011). The larger droplets produced when Tween 20 is used as surfactant can lead to lower nanoemulsion stability as measured in terms of particle size. These larger droplets result in lower specific surface areas, being more susceptible to surface area fluctuations, increasing creaming and coalescence phenomena (Tadros, 2013).

\subsection{Selection of the most suitable process conditions}

The results showed that for anionic nanoemulsions the smallest sizes were achieved using the highest pressure and highest number of cycles tested, 20,000 Psi and 30 cycles of homogenization (Table 2). In fact, a higher homogenization pressure and number of cycles increase the specific energy delivered by the high-pressure homogenizer, which is responsible for increasing the disruption rates that, in the presence of enough surfactant, will lead to smaller sizes (Donsì et al., 2011). Nevertheless, performing a one-way ANOVA to the obtained size values it was possible to verify that there were no statistically significant differences between the results achieved with 15,000 and 20,000 Psi and between 20 and 30 cycles $(p=0.2269$ ). Being so, it can be concluded that 15,000 Psi and 20 cycles are the most suitable process conditions to carry on the study of creaming during long-term storage. The surfactant concentration and the oil content did not show statistically significant differences in terms of $H_{d}$.

For the cationic nanoemulsions the most influent parameters were surfactant concentration $(p=0.0048)$, followed by ratio between oily and aqueous phases $(p=0.014)$ and pressure $(p=0.0289)$. The increase of surfactant concentration leads to the formation of smaller nanoemulsions, once surfactant adsorption to the interface may lower the interfacial tension, resulting in a higher mechanical resistance to rupture (McClements, 2005). Low oil content leads to the decrease of droplet size; this can be explained by the influence of oil in nanoemulsions viscosity (i.e. low oil content leads to lower viscosity values). Higher viscosities induce a flow resistance in the chamber of the high-pressure homogenizer diminishing the rate and efficiency of droplet disruption, leading to higher mean droplet sizes (McClements, 2005; Troncoso et al., 2012). For the nanoemulsions produced with non-ionic surfactant the significant parameters were the combination of pressure with oil content ( $p=0.0025)$, the surfactant concentration $(p=0.0145)$, pressure $(p=0.0150)$ and the number of cycles $(p=0.0247)$. Here the increase in oil content lead to smaller $H_{d}$ values, contrary to the usual assumption that the increase of the oil content increases $H_{d}$ values due to the influence of oil in the viscosity (McClements, 2005; Troncoso et al., 2012). An increase in oil content results in the increase of the concentration of droplets (i.e. increase of the dispersed phase volume fraction) in an emulsion, which leads to higher frequency of droplet collisions and hence coalescence during emulsification. Also, the viscosity of the emulsion increases, which may change the flow from turbulent to laminar. The presence of more particles results in an increase of the velocity gradient. In turbulent flow, the increase of oil content can induce turbulence depression leading to larger droplet sizes. If the ratio between the surfactant and the continuous phase is constant, an increase in oil content results in a decrease of the surfactant concentration, resulting in larger droplets. Nevertheless, if the ratio between the surfactant concentration and the disperse phase is kept constant, then this is reversed, so at this point it is impossible to draw any conclusions regarding the mechanisms that may come into play (McClements, 2005; Schramm, 2006a; Tadros, 2013).

The selection of the most suitable process conditions for the three surfactants was rather difficult since for each surfactant different process parameters had different responses. However, due to the need to select one formulation for further study the central point formulation was selected (15,000 Psi, 20 cycles, $1 \%$ wt of surfactant and an oil content of $10 \%$ wt). The pressure of 20,000 Psi and the 30 cycles of homogenization were not considered, due to the overheating that these conditions induced to the nanoemulsion solution, that could be harmful for bioactive compounds that are heat sensitive. Also, increasing the pressure and the number of cycles increases the energy consumption, which can increase the 
price of producing nanoemulsions. An oily/aqueous phase volume ratio of $10 \%$ wt was used according to Silva et al. (2011) and Tan and Nakajima (2005). Tan and Nakajima (2005) studied the influence of phase ratios (1:9 and 2:8); as the oil content increases, the available surfactant decreases, reducing the number of molecules able to stabilize the formed droplets, thus favoring coalescence and resulting in higher mean droplet diameters. In addition, higher viscosity of the oily phase leads to a less favorable mixing efficiency (Tan and Nakajima, 2005).

\subsection{Kinetic stability for the selected conditions}

Kinetic stability tests are useful not only to provide information about the short-term stability of nanoemulsions, but also for predicting the long-term stability. The input of high temperature and centrifugal forces increases Brownian motion and allows the dispersed droplets to approach one another (McClements, 2005; Morais Diane and Burgess, 2014; Schramm, 2006b). Information's such as the rate at which the properties of a nanoemulsion change with time (i.e. time dependence) is highly important for food industry (McClements, 2005; Schramm, 2006b; Walstra, 1993). The selected nanoemulsion formulations were studied in terms of centrifugation, heating-cooling cycles, creaming and size stability during storage (35 days) at $4{ }^{\circ} \mathrm{C}$. Creaming and size stability during storage were also evaluated after one year of storage at $4{ }^{\circ} \mathrm{C}$, in the absence of light.

Nanoemulsions produced with anionic, cationic and non-ionic surfactants showed no macroscopic sign of instability phenomena (i.e. creaming or phase separation) after centrifugation. The results showed that the nanoemulsions produced with the anionic, cationic and non-ionic surfactants maintained their mean droplet size and hence were stable even under a centrifugal stress of $5000 \mathrm{~g}$ (Table 5). Results showed that these nanoemulsions under centrifugal tests did not present statistically significant $(p>0.05)$ differences in the values of $H_{d}$ when compared to those maintained at $4{ }^{\circ} \mathrm{C}$ (see Table 5). Similar results were obtained following the heating-cooling tests (Table 5).

For the same process and formulation conditions nanoemulsions produced with SDS achieved lower sizes compared to the cationic or non-ionic surfactant nanoemulsions (Fig. 2). The differences in $H_{d}$ values for the different surfactants can be attributed to the molecular weight $\left(M_{w}\right)$ of the surfactants $\left(M_{w}\right.$ SDS $<M_{w}$ $\mathrm{DTAB}<M_{w}$ Tween 20). In general small molecule surfactants can lead, under similar processing conditions, to smaller $H_{d}$ values (Donsì et al., 2011; Qian and McClements, 2011). Within the homogenizer chambers small molecule surfactants are able to quickly adsorb to the newly formed droplets surface (Qian and McClements, 2011; Troncoso et al., 2012).

The theoretical minimum size of the produced nanoemulsions through high-pressure homogenization was calculated using Eq. (5).

$R_{\min }=(3 * \Gamma * \phi) /\left(c_{s}^{*}(1-\phi)\right)$

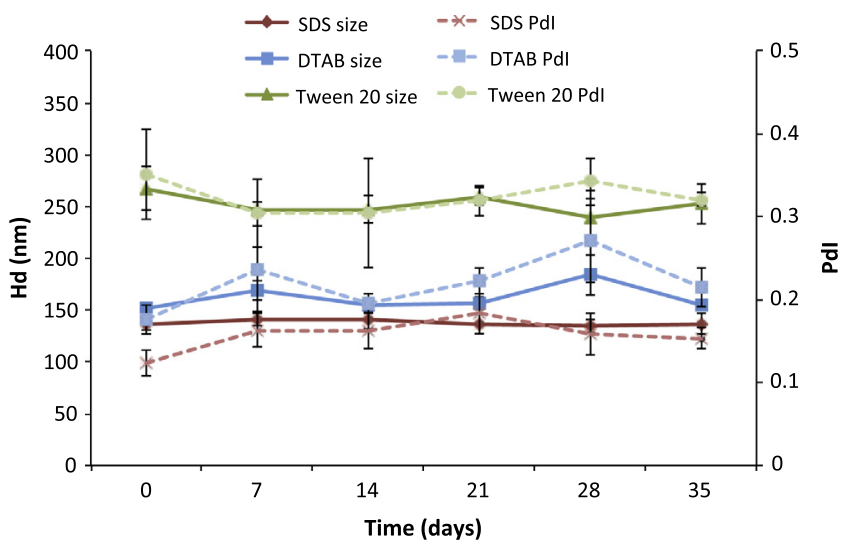

Fig. 2. Hydrodynamic diameter $\left(H_{d}\right)$ and polydispersity index (PdI) during 35 days of storage. Bars indicate standard deviation $(n=3)$. Lines are for readers' guidance and do not represent a model prediction.

Here, $\Gamma$ is the surface load of the surfactant at saturation $\left(\mathrm{kg} / \mathrm{m}^{2}\right), \phi$ is the disperse phase volume (dimensionless), and $C_{s}^{\prime}$ is the concentration of surfactant at the continuous phase $\left(\mathrm{kg} / \mathrm{m}^{3}\right)$ (Troncoso et al., 2012). The surface load of SDS, DTAB and Tween 20 were assumed as 1,1 and $1.5 \mathrm{mg} / \mathrm{m}^{2}$, respectively (Berton-Carabin et al., 2014). The minimum theoretical droplet diameter achieved was $67 \mathrm{~nm}$ for the anionic and cationic nanoemulsions and $100 \mathrm{~nm}$ for the non-ionic nanoemulsion. The experimental mean droplet diameter obtained in our study was $137 \mathrm{~nm}$ for the anionic nanoemulsion, $152 \mathrm{~nm}$ for the cationic nanoemulsion and $223 \mathrm{~nm}$ for the non-ionic nanoemulsion. These results are far from the theoretical ones, which suggests that there was not enough surfactant initially present to cover all droplets, or the homogenizer was incapable of generating enough disruptive forces for the process conditions used (Qian and McClements, 2011; Troncoso et al., 2012). The oil viscosity has an important role in the mean droplet sizes. Troncoso et al. (2012) showed that for more viscous oily phases the high-pressure homogenizer used to prepare the nanoemulsions was incapable of generating sufficiently intensive disruptive forces, while reducing the viscosity of the oil lead to similar results between the experimental work and the theoretical data (Troncoso et al., 2012).

Also the ability to lower oil:water interfacial tension and the faster kinetics of adsorption at the oil:water interface can explain the differences in the mean droplet size (Donsì et al., 2011). A decrease in interfacial tension, while leading to lower droplet sizes, also increases the surface area of nanoemulsions. The properties of colloidal systems' surface are very important and lead to unique physical properties (Schramm, 2006a, 2006b, 2006c). The specific surface area of nanoemulsions, considering $n$ spheres of density $\rho$ and radius $R$ was determined (Eq. (6)) for the selected conditions (Schramm, 2006a).

Table 5

Experimental $H_{d}$ and PdI values for nanoemulsions produced with SDS, Tween 20 and DTAB as surfactants after kinetic stability tests.

\begin{tabular}{|c|c|c|c|c|c|c|}
\hline \multirow[t]{2}{*}{ Treatment } & \multicolumn{2}{|l|}{ SDS } & \multicolumn{2}{|l|}{ DTAB } & \multicolumn{2}{|l|}{ Tween 20} \\
\hline & $H_{d}(\mathrm{~nm})$ & PdI & $H_{d}(\mathrm{~nm})$ & PdI & $H_{d}(\mathrm{~nm})$ & PdI \\
\hline After production & $137.4 \pm 0.6^{\mathrm{a}}$ & $0.128 \pm 0.04^{a}$ & $152.1 \pm 2.1^{\mathrm{a}}$ & $0.178 \pm 0.02^{\mathrm{a}}$ & $223.2 \pm 1.4^{\mathrm{a}}$ & $0.218 \pm 0.01^{a}$ \\
\hline After centrifugation & $134.8 \pm 2.4^{\mathrm{a}}$ & $0.126 \pm 0.03^{a}$ & $155.4 \pm 2.1^{\mathrm{a}}$ & $0.177 \pm 0.01^{a}$ & $225.6 \pm 1.1^{\mathrm{a}}$ & $0.221 \pm 0.02^{a}$ \\
\hline After heating-cooling cycles & $136.3 \pm 3.0^{\mathrm{a}}$ & $0.121 \pm 0.03^{\mathrm{a}}$ & $155.7 \pm 2.6^{\mathrm{a}}$ & $0.176 \pm 0.01^{\mathrm{a}}$ & $221.2 \pm 4.6^{\mathrm{a}}$ & $0.217 \pm 0.01^{\mathrm{a}}$ \\
\hline After 1 year of storage & $138.5 \pm 1.3^{\mathrm{a}}$ & $0.129 \pm 0.05^{a}$ & n.a. & n.a. & n.a. & n.a. \\
\hline
\end{tabular}

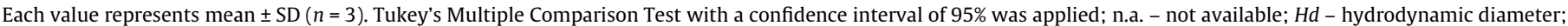
$Z p$ - zeta potential; PdI - polydispersity index.

a Different letters between rows means statistically different. 
$A_{\mathrm{sp}}=3 / \rho R$

The combination of high-pressure homogenization with the intrinsic properties of the anionic surfactant lead to a specific surface area of $44.3 \mathrm{~m}^{2} / \mathrm{g}$ of oil promoting, promoting an increase of ca. 37,000 times of the surface area which was of $0.0012 \mathrm{~m}^{2} / \mathrm{g}$ of oil before the high-pressure homogenization process. Higher specific surface areas (smaller droplets) are less susceptible to surface fluctuations and hence creaming and coalescence are reduced, explaining the high stability of nanoemulsions (Tadros, 2013). As expected, due to the higher droplet sizes, cationic and non-ionic surfactants nanoemulsions had lower specific surface areas. The cationic nanoemulsion had 1.1 times less surface area when compared to the anionic nanoemulsion, achieving a specific surface area of $39.7 \mathrm{~m}^{2} / \mathrm{g}$ of oil. Non-ionic nanoemulsion had 1.9 times less surface area when compared to the anionic nanoemulsion, achieving a specific surface area of $22.6 \mathrm{~m}^{2} / \mathrm{g}$ of oil.

Consumers expect homogenous products, without the undesirable separation of emulsion into oily (opaque and rich in droplets) and aqueous phases (less opaque and without droplets). In order to evaluate emulsion homogeneity, creaming was determined after 1 year of storage at $4{ }^{\circ} \mathrm{C}$. Using Stokes equation, where the terminal settling velocity $(\mathrm{d} x / \mathrm{d} t)$ is proportional to gravity $(g)$ and the square of particle size $(a)$, and inversely proportional to the fluid viscosity $(\eta)$, it is possible to predict the rate of creaming once it is proportional to the settling velocity.

$(\mathrm{d} x / \mathrm{d} t)=\left(2 a^{2}\left(\rho_{2}-\rho_{1}\right) g\right) /(9 \eta)$

Stokes law assumes that emulsions are uncharged and spherical; nevertheless, since the droplets will interact in the case of charged nanoemulsions stabilized with surfactants or polymers (opposite to what is assumed in Stokes' theory), underestimations or overestimations of the terminal velocity are to be expected (Schramm, 2006a). When the emulsions are charged an electrical potential is created; this speeds up the counter-ions and slows down the droplets, although this effect can be quickly dissipated at high enough electrolyte concentrations. Applying a correction factor to the Stokes' law it is possible to determine the settling velocity. Richardson-Zaki equation (Eq. (8)) was applied for this, where $\Phi$ is the dispersed-phase volume fraction $(\Phi<1)$, for particles smaller than $1 \mu \mathrm{m}$ in diameter the exponent found was 5.25 (Barnes and Holbrook, 1993; Schramm, 2006a).

$(\mathrm{d} x / \mathrm{d} t)^{\prime}=(\mathrm{d} x / \mathrm{d} t) *(1-\phi)^{5.25}$

Anionic nanoemulsions showed no macroscopic sign of creaming or phase separation (Table 5); nevertheless, cationic and non-ionic nanoemulsions presented a phase separation due to creaming with a height of 0.25 and $0.5 \mathrm{~cm}$ after 1 year of storage, respectively. The obtained creaming values for the nanoemulsions produced with Tween 20 are in agreement with the predicted values $(0.42 \mathrm{~cm} /$ year $)$; nevertheless, for anionic and cationic nanoemulsions the same behavior was not verified. The predicted value for the cationic nanoemulsion was $0.09 \mathrm{~cm} /$ year, opposing to the obtained value of $0.25 \mathrm{~cm} /$ year. One possible explanation for this discrepancy is that even reducing droplet sizes to $155 \mathrm{~nm}$ this was not enough to overcome the density difference between dispersed and continuous phases. Here the gravitational forces exceed the Brownian motion, creating a concentration gradient that makes the larger droplets quickly move to the top of the container (Schramm, 2006a; Tadros, 2013). For the anionic nanoemulsion the predicted creaming value was $0.07 \mathrm{~cm} /$ year; nevertheless, no macroscopic sign of instability phenomena (i.e. creaming or phase separation) was visible. As the gravity force is proportional to $R^{3}$, if $R$ is reduced 10 times the gravity force is reduced by 1000 times. Below 100 to $200 \mathrm{~nm}$ of droplet size (which also depends on the density difference between the oily and water phases), Brownian motion can exceed the gravity force and therefore creaming is prevented (Tadros, 2013). The smaller size obtained by the anionic nanoemulsions is one of the reasons that allow explaining why these nanoemulsions had higher stability than the cationic and non-ionic nanoemulsions. Also, increasing the surface charge of the droplets can slow down the creaming rate because of the increasing repulsive forces between the droplets (McClements, 2005).

After 1 year of storage at $4{ }^{\circ} \mathrm{C}$, the nanoemulsions produced with cationic and non-ionic surfactants showed visible creaming, presenting a creaming height of 0.25 and $0.5 \mathrm{~cm}$ (data not shown), respectively. The nanoemulsion produced with the anionic surfactant remained kinetically stable, without evidence of creaming and/or phase separation, showing an $H_{d}$ of $138.5 \mathrm{~nm}$.

\subsection{Effect of temperature in size stability}

The thermal stability tests performed to select nanoemulsions (please see topic 3.3.) showed no macroscopic sign of instability phenomena (i.e. creaming or phase separation) after heating the samples from 20 to $80^{\circ} \mathrm{C}$. For this test, $H_{d}$ measurements were performed immediately after nanoemulsions' preparation and following the $30 \mathrm{~min}$ of heating at each temperature $\left(20-80^{\circ} \mathrm{C}\right.$, refer to Table 6). Temperature increase did not have an immediate effect in the characteristics of nanoemulsions, e.g. statistical analysis showed that thermal stress did not provoke significant differences in the mean hydrodynamic diameter (Table 6). This is due the fact that unlike microemulsions, that change their morphology, size and shape as a function of temperature and/or composition, nanoemulsions stability is less sensitive to temperature increase, and this change does not promote a modification in the continuous phase, being the droplet sizes unchangeable (Anton and Vandamme, 2011; Gordon et al., 2014).

\subsection{Energy consumption}

One of the drawbacks of high-energy methods like high-pressure homogenization is the significant amount of energy required during the process (Tadros et al., 2004; Walstra, 1993). It is therefore important to evaluate the interfacial energy (Eq. (1)), the mechanical energy (Eq. (4)) and the \% of energy dissipated into heat. Results showed that mechanical energy exceeded interfacial energy by several orders of magnitude. The interfacial energy values required for the formation of anionic, cationic and non-ionic nanoemulsions were $1.97,1.92$ and $0.91 \mathrm{~J}$, respectively, while the mechanical energy was $2070 \mathrm{MJ}$ for all the nanoemulsions. This means an efficiency of $0.1 \%$, while $99.9 \%$ of the energy was dissipated as heat. These results are in agreement with the results present by Tadros et al. (2004) and Walstra (1993).

\section{Conclusions}

Homogenization pressure, surfactant type and oil concentration were found to be critical to achieve the desired hydrodynamic diameters, polydispersity index and zeta potential of nanoemulsions. Although nanoemulsions were produced at all levels of process and formulation parameters being the parameters' values for the preparation of very stable nanoemulsions with the smallest hydrodynamic diameter: homogenization pressure of 20,000 Psi, 20 cycles of homogenization, $1 \%$ wt of surfactant and $10 \%$ wt of oil. Results showed that differently charged nanoemulsions were kinetically stable during storage, 28 days (for fractional factorial design) and 35 days (for the selected formulations) at $4{ }^{\circ} \mathrm{C}$ in terms of hydrodynamic diameter, polydispersity index and zeta potential. However, the anionic nanoemulsion displayed the highest stability 
Table 6

Experimental $H_{d}$ and PdI values for nanoemulsions produced with SDS, Tween 20 and DTAB as surfactants after thermal stress tests.

\begin{tabular}{|c|c|c|c|c|c|c|}
\hline \multirow[t]{2}{*}{ Treatment } & \multicolumn{2}{|l|}{ SDS } & \multicolumn{2}{|l|}{ DTAB } & \multicolumn{2}{|l|}{ Tween 20} \\
\hline & $H_{d}(\mathrm{~nm})$ & PdI & $H_{d}(\mathrm{~nm})$ & PdI & $H_{d}(\mathrm{~nm})$ & PdI \\
\hline After production & $137.5 \pm 0.2^{\mathrm{a}}$ & $0.128 \pm 0.04^{\mathrm{a}}$ & $152.1 \pm 2.1^{\mathrm{a}}$ & $0.178 \pm 0.02^{\mathrm{a}}$ & $223.2 \pm 1.4^{\mathrm{a}}$ & $0.218 \pm 0.01^{a}$ \\
\hline $20^{\circ} \mathrm{C}$ & $138.1 \pm 0.7^{\mathrm{a}}$ & $0.128 \pm 0.01^{\mathrm{a}}$ & $153.4 \pm 1.1^{\mathrm{a}}$ & $0.174 \pm 0.02^{\mathrm{a}}$ & $223.3 \pm 1.1^{\mathrm{a}}$ & $0.218 \pm 0.01^{a}$ \\
\hline $30^{\circ} \mathrm{C}$ & $139.0 \pm 1.6^{\mathrm{a}}$ & $0.128 \pm 0.02^{\mathrm{a}}$ & $156.2 \pm 1.8^{\mathrm{a}}$ & $0.176 \pm 0.01^{\mathrm{a}}$ & $226.8 \pm 3.2^{\mathrm{a}}$ & $0.224 \pm 0.06^{a}$ \\
\hline $40^{\circ} \mathrm{C}$ & $139.5 \pm 2.2^{\mathrm{a}}$ & $0.129 \pm 0.02^{\mathrm{a}}$ & $157.6 \pm 2.7^{\mathrm{a}}$ & $0.175 \pm 0.02^{\mathrm{a}}$ & $225.5 \pm 2.8^{\mathrm{a}}$ & $0.214 \pm 0.03^{a}$ \\
\hline $50{ }^{\circ} \mathrm{C}$ & $139.0 \pm 1.4^{\mathrm{a}}$ & $0.127 \pm 0.01^{\mathrm{a}}$ & $155.6 \pm 1.7^{a}$ & $0.178 \pm 0.03^{\mathrm{a}}$ & $224.3 \pm 1.4^{a}$ & $0.218 \pm 0.01^{a}$ \\
\hline $60{ }^{\circ} \mathrm{C}$ & $138.2 \pm 0.7^{\mathrm{a}}$ & $0.127 \pm 0.03^{a}$ & $157.5 \pm 2.2^{\mathrm{a}}$ & $0.178 \pm 0.01^{\mathrm{a}}$ & $227.7 \pm 3.6^{\mathrm{a}}$ & $0.217 \pm 0.02^{a}$ \\
\hline $70{ }^{\circ} \mathrm{C}$ & $139.6 \pm 1.9^{a}$ & $0.127 \pm 0.01^{\mathrm{a}}$ & $155.7 \pm 1.4^{\mathrm{a}}$ & $0.179 \pm 0.02^{\mathrm{a}}$ & $226.6 \pm 2.6^{a}$ & $0.219 \pm 0.01^{\mathrm{a}}$ \\
\hline $80^{\circ} \mathrm{C}$ & $139.4 \pm 2.1^{\mathrm{a}}$ & $0.126 \pm 0.02^{\mathrm{a}}$ & $154.8 \pm 1.9^{\mathrm{a}}$ & $0.178 \pm 0.02^{\mathrm{a}}$ & $224.3 \pm 1.6^{\mathrm{a}}$ & $0.219 \pm 0.01^{a}$ \\
\hline
\end{tabular}

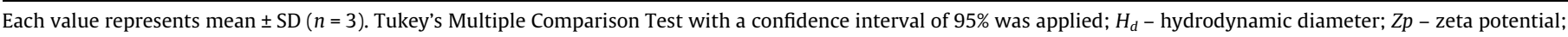
PdI - polydispersity index.

${ }^{a}$ Different letters between rows means statistically different.

against creaming after 1 year of storage. The use of small molecules' surfactants as SDS leads to smaller droplet sizes due to the faster adsorption kinetics to the interface that can reduce size and recoalescence phenomena. The increase of surface charge may slow down the creaming rate, due to the increase of the repulsive forces between droplets. This work showed that it is possible to tailor charged nanoemulsions' size through high-pressure homogenization and the appropriate choice of surfactant.

\section{Acknowledgements}

Hélder D. Silva, and Miguel A. Cerqueira (SFRH/BD/81288/2011, and SFRH/BPD/72753/2010, respectively) are the recipients of a fellowship from the Fundação para a Ciência e Tecnologia (FCT, Portugal). The authors would like to acknowledge to Francesco Donsì and Giovanna Ferrari from Department of Industrial Engineering, University of Salerno for helping in the viscosity and density measurements and to Rui Fernandes from IBMC, University of Porto for assistance in taking the TEM pictures. The authors thank the FCT Strategic Project PEst-OE/EQB/LA0023/2013 and the project "BioInd-Biotechnolog $\mathrm{y}$ and Bioengineering for improved Industrial and Agro-Food processes", REF.NORTE-07-0124- FEDER-000028, co-funded by the Programa Operacional Regional do Norte (ON.2 - O Novo Norte), QREN, FEDER. We also thank to the European Commission: BIOCAPS (316265, FP7/REGPOT-2012-2013.1). The support of EU Cost Action FA1001 is gratefully acknowledged. Also the authors acknowledge Stepan for providing the Neobee 1053 oil.

\section{References}

Abbas, S., Hayat, K., Karangwa, E., Bashari, M., Zhang, X., 2013. An overview of ultrasound-assisted food-grade nanoemulsions. Food Eng. Rev. 5 (3), 139-157.

Acosta, E., 2009. Bioavailability of nanoparticles in nutrient and nutraceutica delivery. Curr. Opin. Colloid Interface Sci. 14, 3-15.

Anton, N., Gayet, P., Benoit, J.-P., Saulnier, P., 2007. Nano-emulsions and nanocapsules by the PIT method: an investigation on the role of the temperature cycling on the emulsion phase inversion. Int. J. Pharm. 344 (1 2), 44-52.

Anton, N., Vandamme, T., 2011. Nano-emulsions and micro-emulsions: clarifications of the critical differences. Pharm. Res. 28 (5), 978-985.

AquaNova, 2013a. <http://www.aquanova.de/media/public/pdf_produkteunkosher/ NovaSOL_beverage.pdf $>$.

AquaNova, 2013b. <http://www.aquanova.de/media/public/pdf_produkteunkosher/ NovaSOL BCS.pdf>.

Barnes, H.A., Holbrook, S.A., 1993. High concentration suspensions. In: Shamlou, P.A. (Ed.), Preparation and Properties. Processing of Solid-Liquid Suspensions. Butterworth-Heinemann, Boston, pp. 222-245.

Berton-Carabin, C.C., Ropers, M.-H., Genot, C., 2014. Lipid oxidation in oil-in-water emulsions: involvement of the interfacial layer. Comprehens. Rev. Food Sci. Food Safe. 13 (5), 945-977.

Brösel, S., Schubert, H., 1999. Investigations on the role of surfactants in mechanical emulsification using a high-pressure homogenizer with an orifice valve. Chem. Eng. Process. 38 (4-6), 533-540.
Cerqueira, M., Pinheiro, A., Silva, H., Ramos, P., Azevedo, M., Flores-López, M., Rivera, M., Bourbon, A., Ramos, Ó., Vicente, A., 2014. Design of bio-nanosystems for oral delivery of functional compounds. Food Eng. Rev. 6 (1-2), 1-19.

Cerqueira, M.A., Bourbon, A.I., Pinheiro, A.C., Silva, H.D., Quintas, M.A.C., Antonio, A.V., 2013. Edible nano-laminate coatings for food applications. Ecosustainable Polymer Nanomaterials for Food Packaging. CRC Press, pp. 221-252.

Cheong, J.N., Tan, C.P., Man, Y.B.C., Misran, M., 2008. $\alpha$-Tocopherol nanodispersions: preparation, characterization and stability evaluation. J. Food Eng. 89 (2), 204 209.

Donsì, F., Sessa, M., Ferrari, G., 2011. Effect of emulsifier type and disruption chamber geometry on the fabrication of food nanoemulsions by high pressure homogenization. Ind. Eng. Chem. Res. 51 (22), 7606-7618.

EFSA, 2010. Call for scientific data on food additives permitted in the EU and belonging to the functional classes of emulsifiers, stabilisers and gelling agents.

Esquena, J., Sankar, Solans, C., 2003. Highly concentrated W/O emulsions prepared by the PIT method as templates for solid foams. Langmuir 19 (7), 2983-2988.

FDA, 2014a. CFR - Code of Federal Regulations Title 21.

FDA, 2014b. CFR - Code of Federal Regulations Title 21 - Food and Drugs.

FDA, 2014c. CFR - Code of Federal Regulations Title 21 - indirect food additives: adjuvants, production aids and sanitizers.

Gordon, V., Marom, G., Magdassi, S., 2014. Formation of hydrophilic nanofibers from nanoemulsions through electrospinning. Int. J. Pharm. 478 (1), 172-179.

Guttoff, M., Saberi, A.H., McClements, D.J., 2015. Formation of vitamin D nanoemulsion-based delivery systems by spontaneous emulsification: factors affecting particle size and stability. Food Chem. 171, 117-122.

High Vive, 2013. <http://www.highvive.com/sunactiveiron.htm>.

Improveat, 2014. <http://www.improveat.pt/en/products/>.

Jadhav, A.J., Holkar, C.R., Karekar, S.E., Pinjari, D.V., Pandit, A.B., 2015. Ultrasound assisted manufacturing of paraffin wax nanoemulsions: process optimization. Ultrason. Sonochem. 23, 201-207.

Komaiko, J., McClements, D.J., 2015. Low-energy formation of edible nanoemulsions by spontaneous emulsification: factors influencing particle size. J. Food Eng. $146,122-128$.

Li, Y., Hu, M., Xiao, H., Du, Y., Decker, E.A., McClements, D.J., 2010. Controlling the functional performance of emulsion-based delivery systems using multicomponent biopolymer coatings. Eur. J. Pharm. Biopharm. 76 (1), 38-47.

Li, Y., McClements, D.J., 2011. Inhibition of lipase-catalyzed hydrolysis of emulsified triglyceride oils by low-molecular weight surfactants under simulated gastrointestinal conditions. Eur. J. Pharm. Biopharm. 79 (2), 423-431.

Life Enhancement, $2013<$ http://www.life-enhancement.com/shop/product/nsrnanoresveratrol>.

Liu, W., Sun, D., Li, C., Liu, Q., Xu, J., 2006. Formation and stability of paraffin oil-inwater nano-emulsions prepared by the emulsion inversion point method. J. Colloid Interface Sci. 303 (2), 557-563.

Malvern, I., 2011. Dynamic light scattering common terms defined, in: Instruments, M. (Ed.), Worcestershire, UK.

Mason, T.G., Wilking, J.N., Meleson, K., Chang, C.B., Graves, S.M., 2006. Nanoemulsions: formation, structure, and physical properties. J. Phys.: Condens. Matter 18 (41), R635.

McClements, D., 2005. Food Emulsions: Principles, Practice, and Techniques, 2nd ed. CRC Press, Boca Raton, Florida.

McClements, D.J., 2002. Lipid-Based Emulsions and Emulsifiers. CRC Press, Food Lipids.

McClements, D.J., 2010. Emulsion design to improve the delivery of functional lipophilic components. Ann. Rev. Food Sci. Technol. 1 (1), 241-269.

Morais Diane, J.M., Burgess, J., 2014. Vitamin E nanoemulsions characterization and analysis. Int. J. Pharm. 465 (1-2), 455-463.

NanoSinergy, 2013. <http://sprayforlife.com/>.

NutraLease, 2011. <http://www.nutralease.com/Nutra/Templates/showpage.asp? $\mathrm{DBID}=1$ \&LNGID=1\&TMID=84\&FID=767>.

Ozturk, B., Argin, S., Ozilgen, M., McClements, D.J., 2014. Formation and stabilization of nanoemulsion-based vitamin E delivery systems using natural surfactants: Quillaja saponin and lecithin. J. Food Eng. 142, 57-63. 
Pinheiro, A.C., Lad, M., Silva, H.D., Coimbra, M.A., Boland, M., Vicente, A.A., 2013. Unravelling the behaviour of curcumin nanoemulsions during in vitro digestion: effect of the surface charge. Soft Matter 9 (11), 3147-3154.

Qian, C., McClements, D.J., 2011. Formation of nanoemulsions stabilized by model food-grade emulsifiers using high-pressure homogenization: factors affecting particle size. Food Hydrocolloids 25 (5), 1000-1008.

Rao, J., McClements, D.J., 2012. Lemon oil solubilization in mixed surfactant solutions: rationalizing microemulsion \& nanoemulsion formation. Food Hydrocolloids 26 (1), 268-276.

Rao, J., McClements, D.J., 2013. Optimization of lipid nanoparticle formation for beverage applications: influence of oil type, cosolvents, and cosurfactants on nanoemulsion properties. J. Food Eng. 118 (2), 198-204.

Sari, T.P., Mann, B., Kumar, R., Singh, R.R.B., Sharma, R., Bhardwaj, M., Athira, S., 2015. Preparation and characterization of nanoemulsion encapsulating curcumin. Food Hydrocolloids 43, 540-546.

Schramm, L.L., 2006a. Dispersion and dispersed species characterization. Emulsions, Foams, and Suspensions. Wiley-VCH Verlag GmbH \& Co. KGaA, pp. 13-51.

Schramm, L.L., 2006b. Interfacial energetics. Emulsions, Foams, and Suspensions. Wiley-VCH Verlag GmbH \& Co. KGaA, pp. 53-100.

Schramm, L.L., 2006c. Introduction. Emulsions, Foams, and Suspensions. Wiley-VCH Verlag GmbH \& Co. KGaA, pp. 1-12.

Shafiq, S., Shakeel, F., Talegaonkar, S., Ahmad, F.J., Khar, R.K., Ali, M., 2007. Development and bioavailability assessment of ramipril nanoemulsion formulation. Eur. J. Pharm. Biopharm. 66 (2), 227-243.

Shemen Industries, 2013. <http://www.nutritionaloutlook.com/article/bigpotential>.

Silva, H., Cerqueira, M., Vicente, A., 2012. Nanoemulsions for food applications: development and characterization. Food Bioprocess Technol. 5 (3), 854-867.
Silva, H.D., Cerqueira, M.A., Souza, B.W.S., Ribeiro, C., Avides, M.C., Quintas, M.A.C., Coimbra, J.S.R., Carneiro-da-Cunha, M.G., Vicente, A.A., 2011. Nanoemulsions of $\beta$-carotene using a high-energy emulsification-evaporation technique. J. Food Eng. 102 (2), 130-135.

Solans, C., Solé, I., 2012. Nano-emulsions: formation by low-energy methods. Curr. Opin. Colloid Interface Sci. 17 (5), 246-254.

Solgar, 2013. <http://www.solgar.com/SolgarProducts/Nutri-Nano-CoQ-10-AlphaLipoic-Acid-Softgels.htm>.

Song, B., Springer, J., 1996. Determination of interfacial tension from the profile of a pendant drop using computer-aided image processing: 2. Experimental. J Colloid Interface Sci. 184 (1), 77-91.

Sood, S. Jain, K. Gowthamarajan, K., 2014. Optimization of curcumin nanoemulsion for intranasal delivery using design of experiment and its toxicity assessment. Colloids Surf., B 113, 330-337.

Stang, M., Schuchmann, H., Schubert, H., 2001. Emulsification in high-pressure homogenizers. Eng. Life Sci. 1 (4), 151-157.

Tadros, T., Izquierdo, P., Esquena, J., Solans, C., 2004. Formation and stability of nano-emulsions. Adv. Colloid Interface Sci. 108-109, 303-318.

Tadros, T.F., 2013. Emulsion Formation, Stability, and Rheology. Emulsion Formation and Stability. Wiley-VCH Verlag GmbH \& Co. KGaA, pp. 1-75.

Tan, C.P., Nakajima, M., 2005. $\beta$-Carotene nanodispersions: preparation, characterization and stability evaluation. Food Chem. 92 (4), 661-671.

Troncoso, E., Aguilera, J.M., McClements, D.J., 2012. Influence of particle size on the in vitro digestibility of protein-coated lipid nanoparticles. J. Colloid Interface Sci. 382 (1), 110-116.

Walstra, P. 1993. Principles of emulsion formation. Chem. Eng Sci. 48 (2), 333-349.

Wild Flavors Inc., 2013. <http://www.wildflavors.com/NA-EN/assets/File/1Ins_ Colors_Emulsions_1_pdf >. 